\title{
Randomized, Placebo-Controlled Trial of Transdermal Rivastigmine for the Treatment of Encephalopathy in Liver Cirrhosis (TREC Trial)
}

\author{
Patrick P. Basu' ${ }^{1,2}$, Niraj James Shah ${ }^{3}$, Mark M. Aloysius' ${ }^{2}$, Robert S. Brown' \\ ${ }^{1}$ Columbia University College of Physicians \& Surgeons, New York, USA \\ ${ }^{2}$ King's County Hospital, New York, USA \\ ${ }^{3}$ James J. Peters VA Medical Center, New York, USA \\ Email: mark.aloysius5@gmail.com
}

Received 17 April 2014; revised 30 May 2014; accepted 15 June 2014

Copyright (C) 2014 by authors and Scientific Research Publishing Inc. This work is licensed under the Creative Commons Attribution International License (CC BY). http://creativecommons.org/licenses/by/4.0/ cC) (7) Open Access

\section{Abstract}

Objectives: Cognitive dysfunction in patients with hepatic encephalopathy (HE) may be caused by alterations in cholinergic neurotransmission. The objective of the study was to evaluate the efficacy and safety of transdermal rivastigmine in improving cognitive function in patients with overt HE. Design: Randomized, controlled pilot study in which patients with grade 2 or 3 HE were treated with lactulose and randomized to receive either transdermal rivastigmine or placebo for 21 days. The modified encephalopathy scale (MES), object recognition test (ORT), trail test (TT), and serum ammonia were assessed at baseline weekly. Electroencephalography was performed at baseline and the final week of the study. Results: Patients were treated with lactulose $(20 \mathrm{~g} / 30 \mathrm{~mL}$ three times per day) and either transdermal rivastigmine $(4.6 \mathrm{mg} / \mathrm{d} ; n=15)$ or placebo $(n=15)$. Transdermal rivastigmine significantly improved MES, ORT, and TT results compared with placebo $(P \leq 0.0001$ at all 3 weeks for all 3 assessments). Serum ammonia improved in both treatment groups, although there was significantly greater improvement with placebo than rivastigmine after 2 weeks of treatment $(P<0.03)$. There were no differences in electroencephalography results between treatment groups. Conclusions: Transdermal rivastigmine with concomitant lactulose significantly improved cognitive-function in patients with overt HE.

What is already known about this subject?

- Current approaches to the management of HE are primarily designed to reduce the levels of ammonia and other gut-derived toxins.

- Traditional strategies for HE treatment have included non-absorbable disaccharides (to decrease bowel transit time) or rifamixin (non-absorbable antibiotics to reduce ammoniogenic flora). 
- No transdermal cholinomimetic agents have been used with oral lactulose to date, in HE. What are the new findings?

- Transdermal rivastigmine is safe for use in patients with grade 2 \& $3 \mathrm{HE}$.

- Transdermal rivastigmine in combination with oral lactulose in this study is far superior to lactulose alone in improving cognitive function.

How might it impact on clinical practice in the foreseeable future?

- Transdermal rivastigmine in combination with oral lactulose can be used safely in clinical practice.

- Transdermal rivastigmine in combination with oral lactulose is efficacious in improving cognitive function in moderate HE (grade 2/3).

Further validation through large randomized clinical trials is required before this is adopted in universal clinical practice of treating HE.

\title{
Keywords
}

\author{
Acetylcholinesterase, Cholinergic Function, Hepatic Encephalopathy, Rivastigmine
}

\section{Introduction}

Hepatic encephalopathy (HE) is a neuropsychiatric complication of acute and chronic liver disease [1] that contributes to the mortality of patients with end-stage liver disease [2]. This potentially reversible condition [3] is characterized by a variety of symptoms, including changes in consciousness and cognition [4]. The precise pathogenic mechanisms leading to HE are unknown but are primarily believed to involve the accumulation of ammonia and other gut-derived toxins [5]. Such current approaches to the management of HE are primarily designed to reduce the levels of ammonia and other gut-derived toxins using therapies such as non-absorbable disaccharides (e.g., lactulose) and non-systemic antibiotics (e.g., rifaximin) [6].

Accumulation of ammonia and gut-derived toxins can lead to a variety of pathogenic changes, including alterations in neurotransmitter signaling systems [3] [6]. While the changes in neurotransmitter systems may be complex and heterogenous, [7] upregulation of the inhibitory GABAergic and serotonergic pathways and impairment of the excitatory glutamatergic and catecholaminergic pathways have been hypothesized to be involved in the pathogenesis of $\mathrm{HE}$ [6]. Alterations in the cholinergic neurotransmitter system have also been observed in both animal models of HE [8]-[12] and in postmortem brain tissue samples from patients with HE [7] [8] [13]. For example, the level of acetylcholine (ACh) and the neurotransmitter of the cholinergic system, were reduced, and the activity of acetylcholinesterase (AChE), the enzyme that hydrolyzes ACh, was increased in brain extracts from rats with experimentally induced HE [8]. Similarly, the activity of AChE was higher in postmortem brain samples from patients with HE compared with controls without cirrhosis [8]. Given the importance of cholinergic neurotransmission in cognitive function and consciousness [14] alterations such as those, which disrupt cholinergic signaling, may explain the deficits in cognitive function and consciousness observed in patients with HE. Thus, therapies that may restore cholinergic balance may be useful in the treatment of patients with HE.

Rivastigmine is a reversible AChE inhibitor used to treat dementia associated with Alzheimer's disease (AD) and Parkinson's disease [15]. Rivastigmine significantly improved learning in a rat model of HE [8], but, to date, there have been no reports of rivastigmine improving cognitive function in patients with HE. However, case reports of patients with HE treated with the AChE inhibitors neostigmine (with polyethylene glycol [PEG]) [16] [17] and physostigmine [18] have suggested that this class of drugs may be effective for the treatment of HE in humans. The objective of this randomized, placebo-controlled pilot study was to determine the efficacy and safety of transdermal rivastigmine for improving cognitive function in patients with grade 2 or $3 \mathrm{HE}$.

\section{Methods}

This was a randomized, placebo-controlled pilot study conducted between 2009 and 2010. The protocol was approved by the institutional review board at Finestein Institute. The study was performed in accordance with the Declaration of Helsinki, the International Conference on Harmonisation Good Clinical Practice Guidelines, and 
applicable local laws and regulations. Signed informed consent was obtained for each patient before study enrollment.

\subsection{Patient Population}

Patients were eligible if they had grade 2 or 3 HE. Hepatic encephalopathy grades were based on clinical and electroencephalographic criteria [19] [20]. Grade $2 \mathrm{HE}$ was characterized by the presence of lethargy, inappropriate behavior, disorientation, asterixis, abnormal reflexes, and abnormalities on electroencephalogram (EEG) (i.e., slowing, triphasic waves). Patients with grade $3 \mathrm{HE}$ exhibited somnolence (but were rousable), loss of meaningful communication, asterixis, abnormal reflexes, and slowing, triphasic waves on EEG. Exclusion criteria included the recent or current use of antihistamines, metoclopramide, benzodiazepines, cannabinoids, or narcotics; acute gastrointestinal bleeding; sepsis; toxic metabolic syndrome; renal failure; and infection with human immunodeficiency virus. Urine was screened before the study and then weekly to detect the use of narcotics, benzodiazepines, and cannabinoids.

\subsection{Treatments}

Eligible patients were treated with oral lactulose ( $20 \mathrm{~g} / 30 \mathrm{~mL}$ three times daily) and randomized to receive either the transdermal rivastigmine patch (Exelon ${ }^{\circledR}$ Patch; Novartis Pharmaceuticals Corporation, East Hanover, NJ; $4.6 \mathrm{mg} / \mathrm{d}$ ) or a placebo patch for 21 days. Randomization was performed by electronic randomization. Dietary animal protein intake was restricted to $50 \mathrm{~g} / \mathrm{d}$. The use of oral or intravenous antibiotics was prohibited during the 21 days of the study.

\subsection{Assessments}

Hepatic encephalopathy was assessed using the modified encephalopathy score (MES), a measure of memory loss, confusion, sleep disturbance, and comprehension with a minimum score of 4 (mild HE) and a maximum score of 12 (severe HE; Table 1). All questions for the MES were administered in the patient's native language. Psychometric testing was performed using the trail test (TT) [21] and the object recognition test (ORT). The MES, TT, ORT, and serum ammonia levels were assessed at baseline and then weekly. Electroencephalography was performed at baseline and during the final week of the study. Safety was assessed

\subsection{Statistical Analysis}

T-test was used to compare transdermal rivastigmine to placebo by calculating $p$ values for statistical significance (defined as $P \leq 0.05$ ) using SPSS version 20 for mac.

\section{Results}

\subsection{Patients and Baseline Characteristics}

A total of 72 patients were screened (Figure 1). Of 12 who did not participate 8 did not meet the inclusion criteria and 4 declined to participate after signing the consent. Thirty patients were randomized; 15 received the transdermal rivastigmine patch and 15 received a placebo patch.

The demographics of the treatment groups were similar with respect to age, sex, and body mass index (Table 2). There were more black patients and fewer white and Hispanic patients in the transdermal rivastigmine group

Table 1. Modified encephalopathy scoring system ${ }^{\mathrm{a}}$.

\begin{tabular}{cccc}
\hline Variable & Mild (1 point) & Moderate (2 points) & Severe (3 points) \\
\hline Memory loss & Past (30 days) & Recent past (7 days) & Recent (24 hours) \\
Confusion & Not confused & Mildly confused & Very confused \\
Sleep disturbance & Mild (changes in sleep pattern) & Moderate (fragmented sleep pattern) & Severe (less than 2 hours of sleep/night) \\
Comprehension & Well aware of surroundings & Impaired awareness of surroundings & Oblivious of surroundings \\
\hline
\end{tabular}

a Patients are rated from 1 to 3 for each variable, and scores are added to calculate the modified encephalopathy score. Total score can range from 4 (mild) to 12 (severe). 


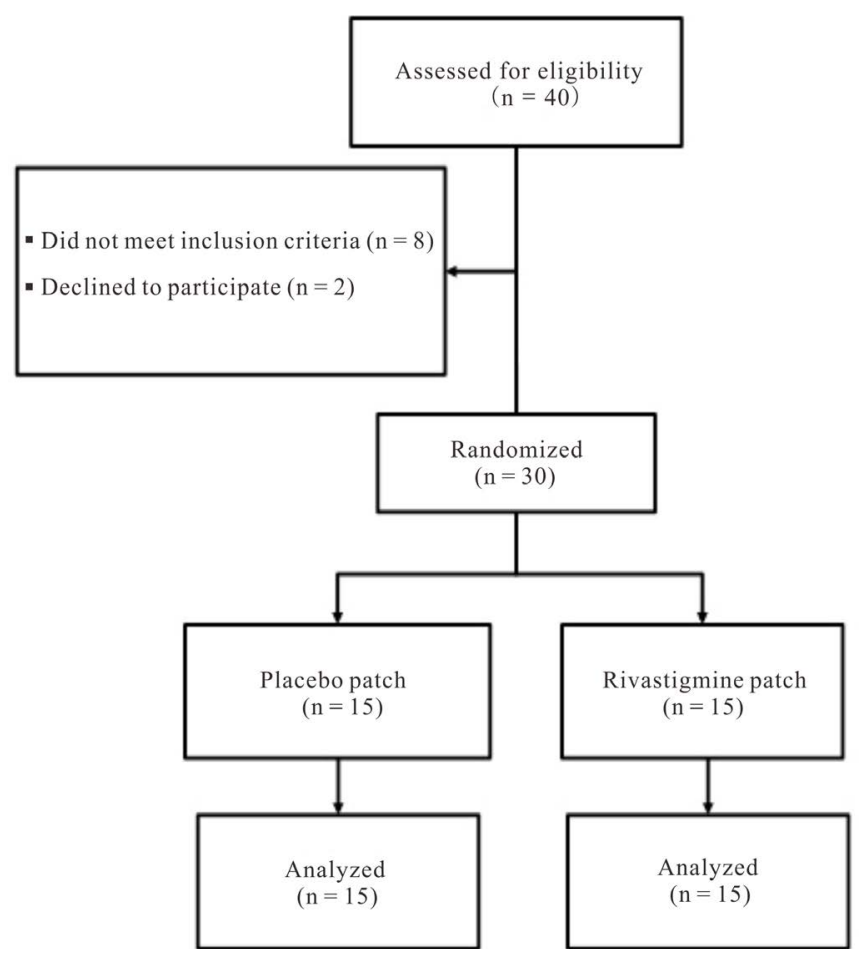

Figure. 1. Patient disposition.

than in the placebo group. Baseline disease characteristics were similar between the treatment groups with respect to model for end-stage liver disease score, EEG, MES, and performance on the TT and ORT (Table 2). More patients in the placebo group had alcoholic liver disease (40\%) compared with the transdermal rivastigmine group (13\%). Serum ammonia levels were slightly but significantly higher in the transdermal rivastigmine group compared with the placebo group $(P=0.03)$.

\subsection{Modified Encephalopathy Score}

Transdermal rivastigmine resulted in a significantly lower mean MES compared with placebo within 1 week of treatment ( 7 vs 10, respectively; $P<0.0001$; Figure 2). Though the mean MES of both treatment groups leveled off during weeks 2 and 3, the significant effect of transdermal rivastigmine compared with placebo on the mean MES was sustained at weeks 2 and 3 of the study $(P<0.0001$ at both weeks). These results suggest that transdermal rivastigmine was significantly more effective than placebo at improving the degree of cognitive dysfunction in HE in patients receiving concomitant lactulose.

\subsection{Psychometric Tests}

Patients who received transdermal rivastigmine had significantly better performance on both the ORT (Figure 3(a)) and TT (Figure 3(b)) during weeks 1 through 3 of the study compared with those who received placebo ( $P$ $=0.0001$ at all 3 time points for both tests). In patients treated with transdermal rivastigmine, ORT results (45 seconds) were normal ( $<55$ seconds) within 1 week of treatment with transdermal rivastigmine. The improvement in the ORT with transdermal rivastigmine was sustained as scores remained normal during weeks 2 and 3 of treatment (35 and 30 seconds, respectively). In contrast, in patients who received placebo, ORT results only slightly improved from 125 seconds at baseline to 98 seconds after 1 week and actually worsened during weeks 2 and 3 of the study (101 and 109 seconds, respectively).

Results of the TT were also substantially improved in patients who received transdermal rivastigmine, decreasing from a baseline score of 161 seconds to 90 seconds and 92 seconds after 1 and 2 weeks of treatment, respectively. By the third week of treatment, TT results ( 65 seconds) approached normal ( $<60$ seconds). In contrast, TT results in the placebo group only slightly improved from 158 seconds at baseline to 154, 134, and 146 
Table 2. Patient demographics and baseline disease characteristics.

\begin{tabular}{lcc}
\hline Characteristic & Placebo (n= 15) & $\begin{array}{c}\text { Transdermal rivastigmine } \\
(\mathbf{n}=\mathbf{1 5})\end{array}$ \\
\hline Mean age (range), y & $56(50-65)$ & $56(51-61)$ \\
Male, n (\%) & $10(67)$ & $9(60)$ \\
Race, n (\%) & \\
White & $6(40)$ & $1(7)$ \\
Black & $3(20)$ & $11(73)$ \\
Hispanic & $6(40)$ & $3(20)$ \\
Mean BMI (range) & $28(26-30)$ & $28(26-30)$ \\
Cirrhosis etiology, n (\%) & & \\
Alcohol & $6(40)$ & $2(13)$ \\
Hepatitis B & $1(7)$ & $3(20)$ \\
Hepatitis C & $5(33)$ & $7(47)$ \\
Nonalcoholic steatohepatitis & $2(13)$ & $1(7)$ \\
Other & $1(7)$ & $2(13)$ \\
HE grade, n (\%) & & 8 \\
2 & 9 & 7 \\
3 & 6 & $20(18-22)$ \\
Mean MELD score (range) & $20(18-22)$ & $245^{\mathrm{b}}$ \\
Serum ammonia, mol/L ${ }^{\mathrm{a}}$ & 239 & 14 \\
Mean MES & 14 & 125 \\
Mean ORT time, $\mathrm{s}^{\mathrm{c}}$ & 122 & 161 \\
Mean TT time, $\mathrm{s}^{\mathrm{d}}$ & 158 & $10(67)$ \\
EEG, n (\%) & & $2(13)$ \\
Normal & $9(60)$ & $0(20)$ \\
Grade 1 & $2(20)$ & \\
Grade 2 & $1(7)$ & \\
Grade 3 & & \\
\hline
\end{tabular}

BMI, body mass index; EEG, electroencephalogram; HE, hepatic encephalopathy; MELD, model for end-stage liver disease; MES, modified encephalopathy score; ORT, object recognition test; TT, trail test. ${ }^{\mathrm{a}}$ Normal range, $11-32 \mathrm{~mol} / \mathrm{L} .{ }^{\mathrm{b}} \mathrm{P}=0.03$. ${ }^{\mathrm{C}}$ Normal, $<55$ seconds. ${ }^{\mathrm{d}}$ Normal, $<60$ seconds.

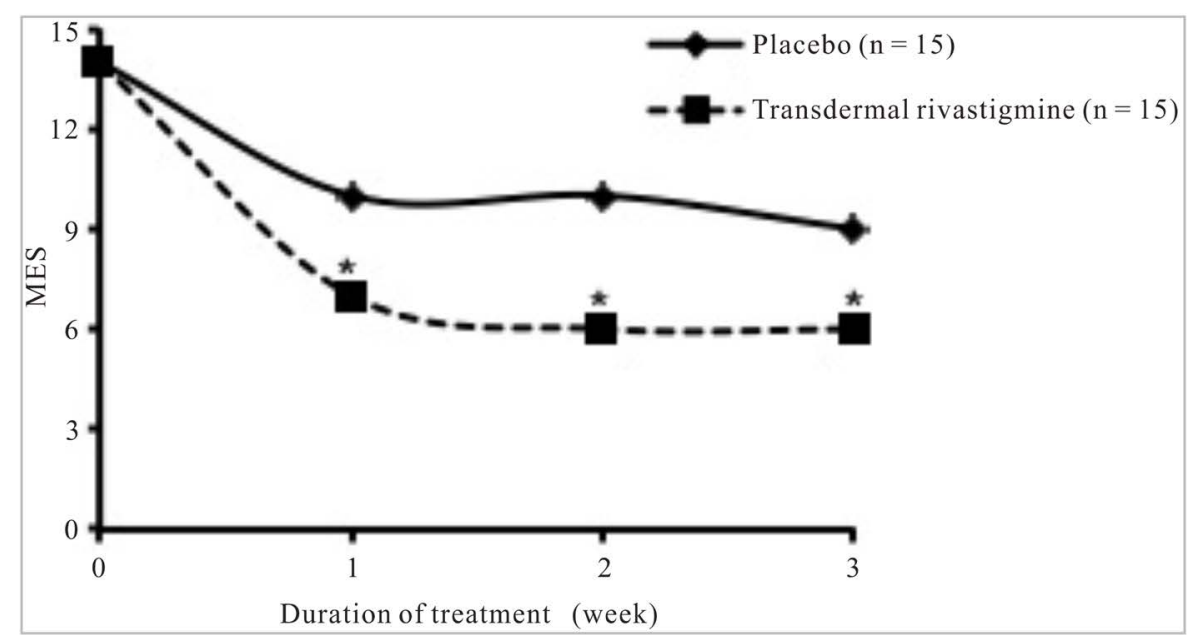

Figure 2. Modified encephalopathy scores over the 3 weeks of treatment with placebo or transdermal rivastigmine. ${ }^{*} P<0.0001$. MES, modified encephalopathy score.

seconds after 1,2 , and 3 weeks of treatment, respectively.

\subsection{Clinical Tests}

For patients in both treatment groups, serum ammonia levels were progressively lower each of the 3 weeks of 


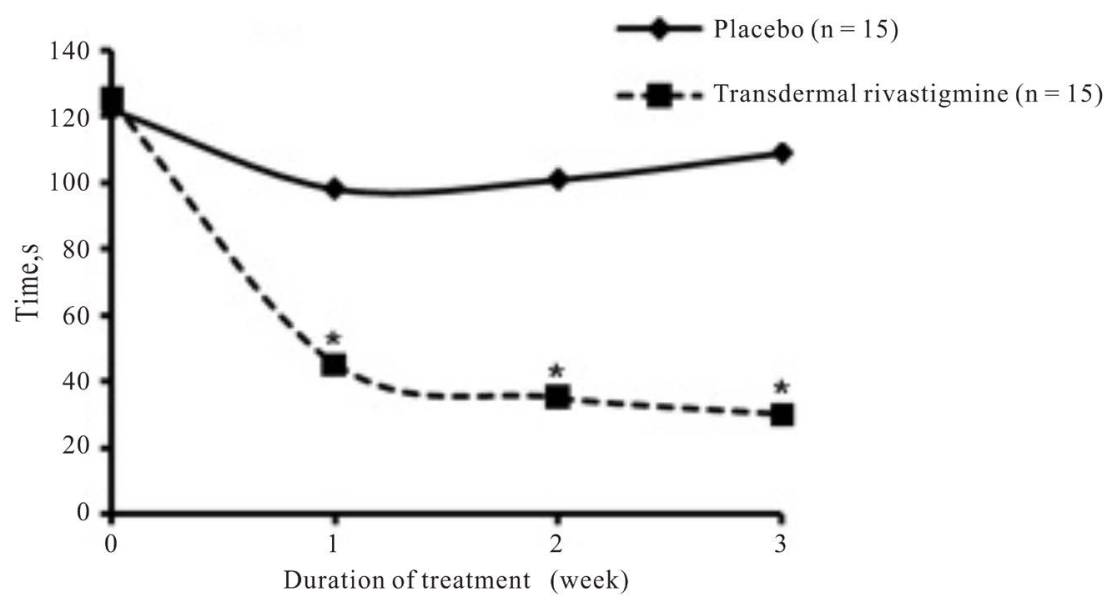

(a)

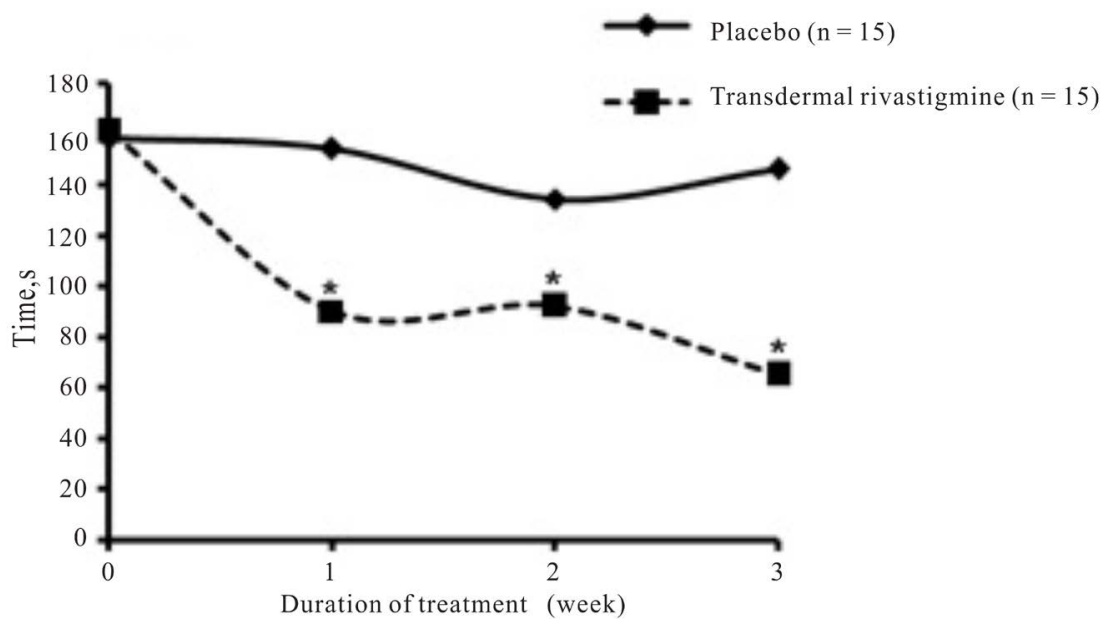

(b)

Figure 3. Psychometric tests. Scores for (a) object recognition test and (b) trail test over the 3 weeks of treatment with placebo or transdermal rivastigmine. Normal scores for object recognition test and trail testare less than 55 seconds and less than 60 seconds, respectively. ${ }^{*} P=0.0001$.

the study compared with baseline (Table 3). However, transdermal rivastigmine did not have a greater effect on serum ammonia levels than placebo. In fact, patients who received placebo had slightly but significantly greater reductions in serum ammonia levels compared with those who received transdermal rivastigmine after 2 weeks of treatment $(P=0.03)$. However, by the third week of treatment, thereduction in serum ammonia was not significantly different between the 2 groups. There were no differences in EEG results between the 2 treatment groups.

\subsection{Safety}

Overall, transdermal rivastigmine was well tolerated, and no reactions were reported at the patch-application site. In the transdermal rivastigmine group, 1 patient (7\%) reported diarrhea and 1 (7\%) reported dry mouth. In the placebo group, 2 patients (13\%) reported diarrhea, 1 (7\%) reported dry mouth, and 1 (7\%) reported urinary retention (Table 4).

\section{Discussion}

The results of this randomized, placebo-controlled pilot study suggest that transdermal rivastigmine significantly improves cognitive function compared with placebo in patients with grade 2 or 3 HE who were receiving con- 
Table 3. Serum ammonia levels.

\begin{tabular}{cccc}
\hline & \multicolumn{2}{c}{ Mean change from baseline, $\mathbf{m o l} / \mathbf{L}$} \\
Treatment week & Placebo $(\mathbf{n}=\mathbf{1 5})$ & $\begin{array}{c}\text { Transdermal rivastigmine } \\
(\mathbf{n}=\mathbf{1 5})\end{array}$ & P value \\
\hline 1 & -92 & -91 & NS \\
2 & -113 & -107 & 0.03 \\
3 & -137 & -131 & 0.05 \\
\hline
\end{tabular}

NS = not significant.

Table 4. Side events.

\begin{tabular}{ccc}
\hline Side Event & Placebo $(\mathbf{n}=\mathbf{1 5})$ & Transdermal rivastigmine $(\mathbf{n}=\mathbf{1 5})$ \\
\hline Diarrhea & 2 & 1 \\
Dry mouth & 1 & 1 \\
Urinary Retention & 1 & 0 \\
\hline
\end{tabular}

improves cognitive function compared with placebo in patients with grade 2 or 3 HE who were receiving concomitant lactulose. These results support those of earlier case reports in which hepatic coma resolved in 3 patients with HE who were treated with other AChE inhibitors [16]-[18]. In 2 of the patients, treatment with neostigmine and PEG electrolyte solution to improve gastric motility and bowel cleansing, respectively, resulted in the resolution of hepatic coma after 2 days [16] [17]. The third patient was treated with physostigmine and immediately regained consciousness after administration of the AChE inhibitor [18].

The observation that inhibition of AChE enhances cognitive function is not unique to HE. Rivastigmine is also used to treat AD and Parkinson's disease dementia. The AChE inhibitors galantamine and donepezil are also used for the treatment of AD. Inhibition of AChE may improve cognitive function in patients with $\mathrm{HE}$, AD, or Parkinson's disease dementia by increasing levels of ACh in the brain and prolonging ACh activity at neural synapses [15]. However, there is mounting evidence that AChE inhibitors may have an antiinflammatory effect [22] which has been observed in patients with AD [23]. Such a mechanism may be relevant to patients with HE because cerebral inflammation has been directly implicated in the pathogenesis of $\mathrm{HE}$ in a rat model of acute liver failure, [24] and systemic inflammation has been implicated in the pathogenesis of HE [25] and the impairment of neuropsychologic function [26] in patients with liver disease. In patients with HE, AChE inhibitors may also increase gastrointestinal motility and thereby enhance the effect of intestinal cathartics such as lactulose and PEG, which were used concomitantly in this study and the previously described case reports, respectively [16]-[18].

While rivastigmine is available in oral and transdermal formulations, the transdermal formulation may be more favorable in general and for patients with HE. First, transdermal rivastigmine generally offers improved tolerability compared with the oral formulation, particularly with respect to gastrointestinal adverse events such as nausea and vomiting [15] [27]. This may be because of the more gradual and smoother increase in absorption of transdermal rivastigmine compared with oral rivastigmine [15]. Second, the ease of dosing of the transdermal patch compared with oral formulations may be advantageous in patients with HE, particularly those with higher grades of HE who may be unable to easily ingest tablets or oral solutions. Lastly, oral rivastigmine is administered twice daily [28] while the patch is applied only once daily [29]. This dosing schedule may provide less interference with daily life. Overall, the advantages of transdermal delivery of rivastigmine may increase patient compliance and satisfaction for patients and caregivers. Indeed, caregivers of patients with AD preferred the transdermal rivastigmine patch compared with the oral capsule in a large ( $n=1059$ caregivers) 24-week study comparing the transdermal and oral formulations of rivastigmine in patients with AD [30].

One limitation to this study is that it was performed using a small population of patients $(\mathrm{N}=30)$. Furthermore, patients in both treatment groups were receiving concomitant lactulose. The finding in this study that there was improvement of MES in the placebo group (albeit to a significantly lesser degree than the transdermal rivastigmine group) suggests that lactulose may have contributed to the improvement in MES observed in this study. However, patients who received placebo did not experience any substantial or sustained benefit in cogni- 
tive function as assessed using the TT and ORT. This suggests that transdermal rivastigmine had an independent effect on cognitive function in patients with grade 2 or $3 \mathrm{HE}$.

Interestingly, despite the improvement in cognitive function with transdermal rivastigmine, there was no improvement in serum ammonia levels with the AChE inhibitor compared with placebo. In fact, after 2 weeks of treatment, serum ammonia levels were reduced to a slightly but significantly greater degree in patients who received placebo compared with those who received transdermal rivastigmine. This suggests that reductions in serum ammonia levels are not clinically relevant in the context of improving the results of psychometric tests such as the TT and ORT. It is notable that serum ammonia levels can be influenced by several factors, including the conditions leading to HE (i.e., precipitating factors), mild exertion by the patient before phlebotomy (including fist clenching), prolonged tourniquet application, and blood-sample drawing technique andstorage [1] [6] [31]-[33]. Thus, results may not be comparable among patients or even between samples taken at different times from an individual patient.

In this study, transdermal rivastigmine with lactulose was safe and well tolerated. Diarrhea, a common adverse event associated with lactulose treatment, [6] was reported by 1 patient who received transdermal rivastigmine and 2 patients who received placebo. Adverse events associated with AChE-inhibitor use were only reported by 1 patient in the transdermal rivastigmine group (dry mouth) and by 2 patients in the placebo group (dry mouth, urinary retention). Further, no skin irritation at the patch-attachment site was reported in this study. The favorable safety and tolerability profile reported here for transdermal rivastigmine in patients with HE is comparable to that reported in patients with $\mathrm{AD}$, although a small percentage of patients with $\mathrm{AD}$ did develop some skin irritation [27].

\section{Conclusion}

In conclusion, although performed with a small number of patients, this randomized, placebo-controlled pilot study demonstrates that transdermal rivastigmine used offlabel with concomitant lactuloseis is safe and effective for improving cognitive function in patients with HE. Large, randomized clinical trials are needed to fully explore the benefits of transdermal rivastigmine in patients with HE. Because of the benefit of transdermal rivastigmine on cognitive function shown here, these trials should be conducted not only in patients with overt HE (grade $\geq 1$ ) but also in patients with minimal HE (grade 0 ) in which the only symptom is cognitive dysfunction measurable only by psychometric or neurophysiologic testing [6].

\section{References}

[1] Häussinger, D. and Schliess, F. (2008) Pathogenetic Mechanisms of Hepatic Encephalopathy. Gut, 57, $1156-1165$.

[2] Stewart, C.A., Malinchoc, M., Kim, W.R. and Kamath, P.S. (2007) Hepatic Encephalopathy as a Predictor of Survival in Patients with End-Stage Liver Disease. Liver Transplantation, 13, 1366-1371. http://dx.doi.org/10.1002/lt.21129

[3] Blei, A.T. and Córdoba, J. (2001) The Practice Parameters Committee of the American College of Gastroenterology. Hepatic Encephalopathy. The American Journal of Gastroenterology, 96, 1968-1976. http://dx.doi.org/10.1111/j.1572-0241.2001.03964.x

[4] Ferenci, P., Lockwood, A., Mullen, K., Tarter, R., Weissenborn, K., Blei, A.T. and Members of the Working Party (2002) Hepatic Encephalopathy_Definition, Nomenclature, Diagnosis, and Quantification: Final Report of the Working Party at the 11th World Congresses of Gastroenterology, Vienna, 1998. Hepatology, 35, 716-721. http://dx.doi.org/10.1053/jhep.2002.31250

[5] Riordan, S.M. and Williams, R. (2010) Gut Flora and Hepatic Encephalopathy in Patients with Cirrhosis. The New England Journal of Medicine, 362, 1140-1142. http://dx.doi.org/10.1056/NEJMe1000850

[6] Mullen, K.D., Ferenci, P., Bass, N.M., Leevy, C.B. and Keeffe, E.B. (2007) An Algorithm for the Management of Hepatic Encephalopathy. Seminars in Liver Disease, 27, 32-48. http://dx.doi.org/10.1055/s-2007-984576

[7] Palomero-Gallagher, N., Bidmon, H.-J., Cremer, M., Schleicher, A., Kircheis, G., Reifenberger, G., et al. (2009) Neurotransmitter Receptor Imbalances in Motor Cortex and Basal Ganglia in Hepatic Encephalopathy. Cellular Physiology and Biochemistry, 24, 291-306. http://dx.doi.org/10.1159/000233254

[8] García-Ayllón, M.S., Cauli, O., Silveyra, M.-X., Rodrigo, R., Candela, A., Compañ, A., et al. (2008) Brain Cholinergic Impairment in Liver Failure. Brain, 131, 2946-2956. http://dx.doi.org/10.1093/brain/awn209

[9] McCandless, D.W., Looney, G.A., Modak, A.T. and Stavinoha, W.B. (1985) Cerebral Acetylcholine and Energy Metabolism Changes in Acute Ammonia Intoxication in the Lower Primate Tupaia glis. Journal of Laboratory and Clinical Medicine, 106, 183-186. 
[10] Méndez, M., Méndez-López, M., López, L., Aller, M.A., Arias, J. and Arias, J.L. (2010) Acetylcholinesterase Activity in an Experimental Rat Model of Type C Hepatic Encephalopathy. Acta Histochemica, 113, 358-362.

[11] Song, G., Dhodda, V.K., Blei, A.T., Dempsey, R.J. and Rao, V.L.R. (2002) GeneChip ${ }^{\circledR}$ Analysis Shows Altered mRNA Expression of Transcripts of Neurotransmitter and Signal Transduction Pathways in the Cerebral Cortex of Portacaval Shunted Rats. Journal of Neuroscience Research, 68, 730-737. http://dx.doi.org/10.1002/jnr.10268

[12] Swapna, I., Sathya SaiKumar, K.V., Murthy, Ch.R.K., Gupta, A.D. and Senthilkumaran, B. (2007) Alterations in Kinetic and Thermotropic Properties of Cerebral Membrane-Bound Acetylcholineesterase during Thioacetamide-Induced Hepatic Encephalopathy: Correlation with Membrane Lipid Changes. Brain Research, 1153, 188-195. http://dx.doi.org/10.1016/j.brainres.2007.02.095

[13] Lal, S., Quirion, R., Lafaille, F., Nair, N.P., Loo, P., Braunwalder, A., et al. (1987) Muscarinic, Benzodiazepine, GABA, Chloride Channel and Other Binding Sites in Frontal Cortex in Hepatic Coma in Man. Progress In NeuroPsychopharmacology \& Biological Psychiatry, 11, 243-250. http://dx.doi.org/10.1016/0278-5846(87)90067-4

[14] Woolf, N.J. and Butcher, L.L. (2010) Cholinergic Systems Mediate Action from Movement to Higher Consciousness. Behavioural Brain Research, 221, 488-498.

[15] Darreh-Shori, T. and Jelic, V. (2010) Safety and Tolerability of Transdermal and Oral Rivastigmine in Alzheimer's Disease and Parkinson's Disease Dementia. Expert Opinion on Drug Safety, 9, 167-176. http://dx.doi.org/10.1517/14740330903439717

[16] Kiba, T., Numata, K. and Saito, S. (2003) Neostigmine and Polyethylene Glycol Electrolyte Solution for the Therapy of Acute Hepatic Encephalopathy with Liver Cirrhosis and Ascites. Hepatogastroenterology, 50, 823-826.

[17] Park, C.H., Joo, Y.E., Kim, H.S., Choi, S.K., Rew, J.S. and Kim, S.J. (2005) Neostigmine for the Treatment of Acute Hepatic Encephalopathy with Acute Intestinal Pseudo-Obstruction in a Cirrhotic Patient. Journal of Korean Medical Science, 20, 150-152. http://dx.doi.org/10.3346/jkms.2005.20.1.150

[18] Kabatnik, M., Heist, M., Beiderlinden, K. and Peters, J. (1999) Hepatic Encephalopathy—A Physostigmine-Reactive Central Anticholinergic Syndrome? European Journal of Anaesthesiology, 16, 140-142.

[19] Stewart, C.A. and Cerhan, J. (2005) Hepatic Encephalopathy: A Dynamic or Static Condition. Metabolic Brain Disease, 20, 193-204. http://dx.doi.org/10.1007/s11011-005-7207-x

[20] Riordan, S.M. and Williams, R. (1997) Treatment of Hepatic Encephalopathy. The New England Journal of Medicine, 337, 473-479. http://dx.doi.org/10.1056/NEJM199708143370707

[21] Conn, H.O. (1977) Trailmaking and Number-Connection Tests in the Assessment of Mental State in Portal Systemic Encephalopathy. The American Journal of Digestive Diseases, 22, 541-550. http://dx.doi.org/10.1007/BF01072510

[22] Tabet, N. (2006) Acetylcholinesterase Inhibitors for Alzheimer's Disease: Anti-Inflammatories in Acetylcholine Clothing! Age Ageing, 35, 336-338. http://dx.doi.org/10.1093/ageing/afl027

[23] Reale, M., Iarlori, C., Gambi, F., Lucci, I., Salvatore, M. and Gambi, D. (2005) Acetylcholinesterase Inhibitors Effects on Oncostatin-M, Interleukin- $1 \beta$ and Interleukin-6 Release from Lymphocytes of Alzheimer's Disease Patients. Experimental Gerontology, 40, 165-171. http://dx.doi.org/10.1016/j.exger.2004.12.003

[24] Jiang, W., Desjardins, P. and Butterworth, R.F. (2009) Cerebral Inflammation Contributes to Encephalopathy and Brain Edema in Acute Liver Failure: Protective Effect of Minocycline. Journal of Neurochemistry, 109, 485-493. http://dx.doi.org/10.1111/j.1471-4159.2009.05981.X

[25] Shawcross, D.L., Wright, G., Damink, S.W.M.O. and Jalan, R. (2007) Role of Ammonia and Inflammation in Minimal Hepatic Encephalopathy. Metabolic Brain Disease, 22, 125-138. http://dx.doi.org/10.1007/s11011-006-9042-1

[26] Shawcross, D.L., Davies, N.A., Williams, R. and Jalan, R. (2004) Systemic Inflammatory Response Exacerbates the Neuropsychological Effects of Induced Hyperammonemia in Cirrhosis. Journal of Hepatology, 40, 247-254. http://dx.doi.org/10.1016/j.jhep.2003.10.016

[27] Winblad, B., Cummings, J., Andreasen, N., Grossberg, G., Onofrj, M., Sadowsky, C., et al. (2007) A Six-Month Double-Blind, Randomized, Placebo-Controlled Study of a Transdermal Patch in Alzheimer's Disease-Rivastigmine Patch versus Capsule. International Journal of Geriatric Psychiatry, 22, 456-467. http://dx.doi.org/10.1002/gps.1788

[28] (2006) Exelon. Novartis Pharmaceuticals Corporation, East Hanover.

[29] (2009) Exelon Patch. Novartis Pharmaceuticals Corporation, East Hanover.

[30] Winblad, B., Kawata, A.K., Beusterien, K.M., Thomas, S.K., Wimo, A., Lane, R., et al. (2007) Caregiver Preference for Rivastigmine Patch Relative to Capsules for Treatment of probable Alzheimer's Disease. International Journal of Geriatric Psychiatry, 22, 485-491. http://dx.doi.org/10.1002/gps.1806

[31] Elgouhari, H.M. and O'Shea, R. (2009) What Is the Utility of Measuring the Serum Ammonia Level in Patients with Altered Mental Status? Cleveland Clinic Journal of Medicine, 76, 252-254. http://dx.doi.org/10.3949/ccjm.76a.08072

[32] Howanitz, J.H., Howanitz, P.J., Skrodzki, C.A. and Iwanski, J.A. (1984) Influences of Specimen Processing and Sto- 
rage Conditions on Results for Plasma Ammonia. Clinical Chemistry, 30, 906-908.

[33] Barsotti, R.J. (2001) Measurement of Ammonia in Blood. The Journal of Pediatrics, 138, S11-S20. http://dx.doi.org/10.1067/mpd.2001.111832 